\title{
SOME REMARKS ON RECONSTRUCTION FROM LOCAL WEIGHTED AVERAGES
}

\author{
P. DEVARAJ
}

\begin{abstract}
We solve the convolution equation of the type $f \star \mu=g$, where $f \star \mu$ is the convolution of $f$ and $\mu$ defined by $(f \star \mu)(x)=\int_{\mathbb{R}} f(x-y) d \mu(y), g$ is a given function and $\mu$ is a finite linear combination of translates of an indicator function on an interval.
\end{abstract}

\section{Introduction}

We consider the convolution equation of the following type:

$$
f \star \mu=g
$$

where $g$ is a known continuous function, $\mu$ is a compactly supported measure and $f$ is an unknown continuous function. Delsarte [3] was interested in solving the particular case of equation (1) which is of the type $\frac{1}{\tau} \int_{x-\frac{\tau}{2}}^{x+\frac{\tau}{2}} f(t) d t=g(x)$. In the case when $f$ is an integrable function with compact support van der Pol $[15,16]$ has obtained reconstruction formula using two sided Laplace Transform. But such transform methods can not be used for the case of continuous functions on $\mathbb{R}$. The special case of equation (1), namely $g=0$, was analyzed by many authors citebag,ber1,deva,Ehr1,Ehr2,kah,schwartz,thangavelu,wei,sze on various groups. The solutions (1) for the particular case when $g=0$ are called mean periodic functions. Laurent Schwartz [18] gave an intrinsic characterization of such solutions. The corresponding nonhomogeneous type equation is analysed in [14]for the special case of when $\mu$ is the indicator function on the interval $[-a, a]$. An explicit construction of a solution is given in [17] for the same equation on the three dimensional Euclidean space when $\mu$ is the indicator function of a ball in $\mathbb{R}^{3}$ using plane wave decomposition. When $\mu$ is finitely supported, the equation (1) gets reduced to a non-homogeneous constant coefficient difference equation. Edgar and Rosenblatt [6] have studied the homogeneous equation (ie, when g=0). They have shown that a complex valued function $f$ has linearly independent translates precisely when $f$ does not

2010 Mathematics Subject Classification. Primary: 42A85, 44A35; Secondary: 42A75, 39A12.

Key words and phrases. Deconvolution, functional equations, mean-periodic functions, reconstruction, unsmoothing. 
satisfy a nontrivial homogeneous difference equation. An explicit construction of a solution is given in [4] on $\mathbb{R}$ when $\mu$ is an arbitrary finitely supported measure and $g$ is a continuous function.

Malgrange [13], Ehrenpreis [9], John [10], and Hörmander [11] have studied the convolution equation of the type analogous to equation (1)

$$
P(D) u=f,
$$

where $P(D)$ is a constant coefficient partial differential operator and $f$ is a given function. A criterion was given by Hörmander for the existence of solution $u \in D_{F}^{\prime}(\Omega)$ for an arbitrary $f \in D_{F}^{\prime}(\Omega)$ on an open set $\Omega \subseteq \mathbb{R}^{n}$.

In general, no necessary and sufficient conditions for the existence of solutions of equation (1) are known. One can easily see the following:

(i) Equation (1) has no solution in $C(\mathbb{R})$ when $g$ is a non smooth function and $\mu$ is a compactly supported continuous function.

(ii) If $f_{0}$ is a particular solution of equation (1), then every other solution $f$ can be written as $f=f_{0}+h$, where $h \star \mu=0$.

(iii) If the Fourier-Laplace transform $\hat{\mu}(\lambda)=0$ for some $\lambda \in \mathbb{C}$ and if there exists a solution to equation (1), then there are infinitely many solutions to equation (1).

The methods of [14] can not be extended to the case when $\mu$ is a sum of more than one indicator function. In this paper we analyze the case when $\mu$ is a finite linear combination of the translates of an indicator function on an interval. A solution $f \in C^{r}(\mathbb{R})$ is constructed for every $g \in C^{r+1}(\mathbb{R})$.

\section{Reconstruction Results}

Definition 1 ([4]). We say a compactly supported Borel measure on $\mathbb{R}$ is a discrete Borel measure, if there exists a finite set of distinct real numbers $x_{1}, x_{2}, \ldots, x_{n}$ and nonzero complex constants $c_{1}, c_{2}, \ldots, c_{n}$ such that $\mu(E)=\sum_{i=1}^{n} c_{i} \delta_{x_{i}}(E)$ for every Borel set $E$. The set of all compactly supported discrete Borel measures on $\mathbb{R}$ is denoted by $M_{c d}(\mathbb{R})$.

For $a, b \in \mathbb{R}$, the indicator function on the interval $[a, b]$ is denoted by $\chi_{[a, b]}$ and $\operatorname{LST}\left(\chi_{[a, b]}\right)$ denotes the linear span of the translates of $\chi_{[a, b]}$. The set of all compactly supported regular Borel measures on $\mathbb{R}$ is denoted by $M_{c}(\mathbb{R})$. We note that $L S T\left(\chi_{[a, b]}\right) \subset M_{c}(\mathbb{R})$.

Definition 2. For $f \in C(\mathbb{R})$ and $\mu \in M_{c}(\mathbb{R})$, the convolution of $f$ with $\mu$ is defined as

$$
(f \star \mu)(x)=\int_{\mathbb{R}} f(x-y) d \mu(y) .
$$


When $\mu=\sum_{i=1}^{n} c_{i} \chi_{\left[a_{i}, b_{i}\right]}$, the convolution becomes

$$
(f \star \mu)(x)=\sum_{i=1}^{n} c_{i} \int_{a_{i}}^{b_{i}} f(x-y) d y .
$$

Definition 3. [4] For every real or complex valued function $f$ and discrete measure $\mu=\sum_{i=1}^{n} c_{i} \delta_{x_{i}}$ $\in M_{c d}(\mathbb{R})$, the convolution of $f$ and $\mu$ is defined by

$$
(f \star \mu)(x)=\sum_{i=1}^{n} c_{i} f\left(x-x_{i}\right) .
$$

In [4] the special case $r=0$ of the following lemma is proved. We extend the same for $r>0$ along the lines of [4].

Lemma 1. For $\mu, v \in M_{c d}(\mathbb{R})$ and $g \in C^{r}(\mathbb{R})$, the following hold:

(i) If $\operatorname{supp}(\mu) \subset(-\infty,-\alpha)$ for some $\alpha>0$ and $\operatorname{supp}(g) \subset(-\infty, \beta)$ for some $\beta \in \mathbb{R}$, then there exists $f \in C^{r}(\mathbb{R})$ such that $f \star\left(\delta_{0}+\mu\right)=g$.

(ii) If $\operatorname{supp}(v) \subset(\alpha, \infty)$ for some $\alpha>0$ and $\sup p(g) \subset(\beta, \infty)$ for some $\beta \in \mathbb{R}$, then there exists $f \in C^{r}(\mathbb{R})$ such that $f \star\left(\delta_{0}+v\right)=g$.

Proof. (i) We denote by $\mu^{m}$ the convolution of $\mu$ with itself m-times. As $\operatorname{supp}(\mu) \subset(-\infty,-\alpha)$, we have $\operatorname{supp}\left(\mu^{n}\right) \subset(-\infty,-n \alpha)$. Let $\mu^{n}=\sum_{i=1}^{l} c_{i} \delta_{y_{i}}$. Then

$$
\left(g^{(j)} \star \mu^{n}\right)(x)=\sum_{i=1}^{l} c_{i} g^{(j)}\left(x-y_{i}\right) .
$$

Since $\operatorname{supp}\left(\mu^{n}\right) \subset(-\infty,-n \alpha), y_{i}<-n \alpha$ and hence $x-y_{i}>x+n \alpha>\beta$ for sufficiently large $n$. Therefore for every $x,\left(g^{(j)} \star \mu^{n}\right)(x)=0$ for $n$ sufficiently large and for $0 \leq j \leq r$.

We define

$$
f(x):=g(x)+\sum_{n=1}^{\infty}(-1)^{n}\left(g \star \mu^{n}\right)(x) .
$$

Let us consider the following partial sums:

$$
s_{n}(x)=g(x)+\sum_{k=1}^{n}(-1)^{k}\left(g \star \mu^{k}\right)(x) .
$$

Then

$$
s_{n}^{(j)}(x)=g^{(j)}(x)+\sum_{k=1}^{n}(-1)^{k}\left(g^{(j)} \star \mu^{k}\right)(x) .
$$


We show that the above sequence converges uniformly on every compact set for $0 \leq j \leq r$. For, let K be a compact subset of $\mathbb{R}$. Then $K \subset[a, b]$ for some real numbers $a$ and $b$. Choose $N$ such that $a+n \alpha>\beta$ and $b+n \alpha>\beta$, for $n \geq N$. For $x \geq a, x-y_{i} \geq a+n \alpha>\beta$. Now

$$
s_{n}^{(j)}(x)-s_{m}^{(j)}(x)=\sum_{k=m+1}^{n}(-1)^{k}\left(g^{(j)} \star \mu^{k}\right)(x)=0,
$$

for $n \geq m \geq N$.

This implies that the sequence of functions $\left\{s_{k}^{(j)}(x)\right\}$ is uniformly cauchy on every compact set and hence converges uniformly on every compact set for $0 \leq j \leq r$.

Therefore we get $s_{k}^{(j)}(x)$ converges uniformly to $f^{(j)}(x)$ on every compact set and

$$
f^{(j)}(x):=g^{(j)}(x)+\sum_{n=1}^{\infty}(-1)^{n}\left(g^{(j)} \star \mu^{n}\right)(x)
$$

for $0 \leq j \leq r$. Hence $f^{(r)}$ is continuous and hence $f^{(r)} \in C^{r}(\mathbb{R})$. It is very easy to check that $f \star\left(\delta_{0}+\mu\right)=g$.

(ii) Since $\operatorname{supp}(v) \subset(\alpha, \infty)$, we have $\operatorname{supp}\left(v^{n}\right) \subset(n \alpha, \infty)$. Suppose the representation of $v^{n}$ is of the form: $v^{n}=\sum_{i=1}^{l} d_{i} \delta_{z_{i}}$. Then $\left(g^{(j)} \star v^{n}\right)(x)=\sum_{i=1}^{l} d_{i} g^{(j)}\left(x-z_{i}\right)$. Since $\operatorname{supp}\left(v^{n}\right) \subset$ $(n \alpha, \infty), z_{i}>n \alpha$ and hence $x-z_{i}<x-n \alpha<\beta$ for sufficiently large $n$. Therefore for every $x$, $\left(g^{(j)} \star v^{n}\right)(x)=0$ for $n$ sufficiently large. Hence $g^{(j)}(x)+\sum_{m=1}^{\infty}(-1)^{m}\left(g^{(j)} \star v^{m}\right)(x)$ is a finite sum for every $x$.

We define

$$
f(x):=g(x)+\sum_{m=1}^{\infty}(-1)^{m}\left(g \star v^{m}\right)(x) .
$$

To show

$$
f^{(r)}(x):=g^{(r)}(x)+\sum_{m=1}^{\infty}(-1)^{m}\left(g^{(r)} \star v^{m}\right)(x),
$$

it is sufficient if we show that the partial sums of the series (3) and their derivatives converge uniformly on compact sets. For, let $\mathrm{K}$ be a compact subset of $\mathbb{R}$. Then $K \subset[a, b]$ for some real numbers $a$ and $b$. Choose $N$ such that $b-n \alpha<\beta$, for $n \geq N$. Let us take the partial sums of the series as

$$
t_{k}(x)=g(x)+\sum_{m=1}^{k}(-1)^{m}\left(g \star v^{m}\right)(x) .
$$

For $x \leq b, x-z_{i}<x-n \alpha \leq b-n \alpha$. Choose $N$ such that $b-n \alpha<\beta$ for $n \geq N$. Then

$$
t_{n}^{(j)}(x)-t_{m}^{(j)}(x)=\sum_{k=m+1}^{n}(-1)^{k}\left(g^{(j)} \star v^{k}\right)(x)=0,
$$

for $n \geq m \geq N$ and $0 \leq j \leq r$. This implies that the sequence $\left\{t_{n}^{(j)}(x)\right\}$ is uniformly cauchy and hence converges uniformly on every compact set. Hence we get $t_{n}^{(j)}(x) \rightarrow f^{(j)}(x)$. Therefore $f \in C^{r}(\mathbb{R})$. One easily verifies $f \star\left(\delta_{0}+v\right)=g$. 
Lemma 2. For $\mu=\chi_{[a, b]}$ and $g \in C^{r+1}(\mathbb{R})$, the following hold: If $\sup p(g) \subset(-\infty, \beta)$ or $\sup p(g) \subset$ $(\beta, \infty)$ for some $\beta \in \mathbb{R}$, then there exists $f \in C^{r}(\mathbb{R})$ such that $f \star \mu=g$.

Proof. Case(i): Suppose that $\operatorname{supp}(g) \subset(-\infty, \beta)$.

We can write

$$
f \star \chi_{[a, b]}=f \star \chi_{\left[\frac{a-b}{2}, \frac{b-a}{2}\right]} \star \delta_{\frac{a+b}{2}} .
$$

Define

$$
f_{1}(x)=-\sum_{n=0}^{\infty}\left(g^{\prime} \star \delta_{\frac{a-b}{2}}^{2 n+1}\right)(x) .
$$

We show that the above series converges uniformly on compact sets. For, let $K$ be a compact subset of $\mathbb{R}$. Then $K \subset[c, d]$ for some $c, d \in \mathbb{R}$. Let us take

$$
s_{n}(x)=-\sum_{k=0}^{n}\left(g^{\prime} \star \delta_{\frac{a-b}{2}}^{2 k+1}\right)(x) .
$$

Then

$$
s_{n}^{(j)}(x)=-\sum_{k=0}^{n}\left(g^{(j+1)} \star \delta_{\frac{a-b}{2}}^{2 k+1}\right)(x) .
$$

Now

$$
g^{\prime} \star \delta_{\frac{a-b}{2}}^{2 k+1}(x)=g^{\prime}\left(x+(2 k+1)\left(\frac{b-a}{2}\right)\right) .
$$

Choose $N$ such that $c+(2 k+1)\left(\frac{b-a}{2}\right)>\beta$ for $k \geq N$. Then $g^{(j+1)} \delta_{\frac{a-b}{2}}^{2 k+1}(x)=0$ for $k \geq N$ for all $x \in K$. Therefore $s_{n}^{(j)}(x)-s_{m}^{(j)}(x)=0$ for all $n, m \geq N$, for all $x \in K$ and for $0 \leq j \leq r$. Hence $s_{n}^{(j)}(x) \rightarrow f_{1}^{(j)}(x)$ uniformly on $K$.

Therefore $f_{1}^{j}(x)=-\sum_{n=0}^{\infty} g^{(j+1)} \star \delta_{\frac{a-b}{2}}^{2 n+1}(x)$ and $f_{1} \in C^{r}(\mathbb{R})$. Now

$$
\begin{aligned}
f_{1} \star \chi_{\left[\frac{a-b}{2}, \frac{b-a}{2}\right]} & =-\sum_{n=0}^{\infty}\left(\left(g^{\prime} \star \chi_{\left[\frac{a-b}{2}, \frac{b-a}{2}\right]}\right) \star \delta_{\frac{a-b}{2}}^{2 n+1}\right)(x) \\
& =-\sum_{n=0}^{\infty} \int_{\frac{a-b}{2}}^{\frac{b-a}{2}} g\left(x+(2 n+1)\left(\frac{b-a}{2}\right)-y\right) d y \\
& =\sum_{n=0}^{\infty}\left[g\left(x+(2 n+1)\left(\frac{b-a}{2}\right)-\frac{b-a}{2}\right)-g\left(x+(2 n+1)\left(\frac{b-a}{2}\right)+\frac{b-a}{2}\right)\right] \\
& =g(x) .
\end{aligned}
$$

Define $f(x)=f_{1} \star \delta_{-\left(\frac{a+b}{2}\right)}$. One easily verifies $f \star \chi_{[a, b]}=g$.

Case(ii): Suppose that $\operatorname{supp}(g) \subset(\beta, \infty)$. Now $f \star \chi_{[a, b]}=f \star \chi_{\left[\frac{a-b}{2}, \frac{b-a}{2}\right]} \star \delta_{\frac{a+b}{2}}$. Define $f_{1}(x)=\sum_{n=0}^{\infty} g^{\prime} \star \delta_{\frac{b-a}{2}}^{2 n+1}(x)$. We show that the above series converges uniformly on compact sets. For, let $K$ be a compact subset of $\mathbb{R}$. Then $K \subset[c, d]$ for some $c, d \in \mathbb{R}$. Let $s_{n}(x)=$ $\sum_{k=0}^{n} g^{\prime} \star \delta_{\frac{b-a}{2}}^{2 k+1}(x)$. Then $s_{n}^{(j)}(x)=\sum_{k=0}^{n} g^{(j+1)} \star \delta_{\frac{b-a}{2}}^{2 k+1}(x)$. 
We can write

$$
g \star \delta_{\frac{b-a}{2}}^{2 k+1}(x)=g\left(x-(2 k+1)\left(\frac{b-a}{2}\right)\right) .
$$

Choose $N$ such that $c-(2 k+1)\left(\frac{b-a}{2}\right)<\beta$ for $k \geq N$. Then $g^{(j+1)} \delta_{\frac{b-a}{2}}^{2 k+1}(x)=0$ for $k \geq N$ for all $x \in K$. Therefore $s_{n}^{(j)}(x)-s_{m}^{(j)}(x)=0$ for all $n, m \geq N$, for all $x \in K$ and for $0 \leq j \leq r$. Hence $s_{n}^{(j)}(x) \rightarrow f_{1}^{(j)}(x)$ uniformly on $K$.

Therefore $f_{1}^{j}(x)=\sum_{n=0}^{\infty} g^{(j+1)} \star \delta_{\frac{b-a}{2}}^{2 n+1}(x)$. and $f_{1} \in C^{r}(\mathbb{R})$.

Now

$$
\begin{aligned}
f_{1} \star \chi_{\left[\frac{a-b}{2}, \frac{b-a}{2}\right]} & =-\sum_{n=0}^{\infty} g^{\prime} \star \chi_{\left[\frac{a-b}{2}, \frac{b-a}{2}\right]} \star \delta_{\frac{b-a}{2}}^{2 n+1}(x) \\
& =\sum_{n=0}^{\infty} \int_{\frac{a-b}{2}}^{\frac{b-a}{2}} g\left(x-(2 n+1)\left(\frac{b-a}{2}\right)-y\right) d y \\
& =-\sum_{n=0}^{\infty}\left[g\left(x-(2 n+1)\left(\frac{b-a}{2}\right)-\frac{b-a}{2}\right)-g\left(x-(2 n+1)\left(\frac{b-a}{2}\right)+\frac{b-a}{2}\right)\right] \\
& =g(x) .
\end{aligned}
$$

Define $f(x)=f_{1} \star \delta_{-\left(\frac{a+b}{2}\right)}$. It is easy to check that $f \star \chi_{[a, b]}=g$.

Lemma 3. Let $\mu=\sum_{i=1}^{n} c_{i} \chi_{\left[a_{i}, b_{i}\right]}$ be a finite linear combination of indicator functions on intervals. If there exists $r \in \mathbb{R}$ such that $\frac{b_{i}-a_{i}}{r} \in \mathbb{Z}$, then the following hold:

(i) There exists $g \in \operatorname{LST}\left(\chi_{[a, b]}\right)$ such that $\mu=g$ almost everywhere for some $a, b \in \mathbb{R}$.

(ii) There exits $\mu \in M_{c d}(\mathbb{R})$, such that $\mu=\chi_{[a, b]} \star v$ a.e and $f \star \mu=f \star \chi_{[a, b]} \star v$ for all $f \in C(\mathbb{R})$.

Proof. (i) Let $\frac{b_{i}-a_{i}}{r}=m_{i}$. Then $\chi_{\left[a_{i}, b_{i}\right]}=\sum_{j=1}^{m_{i}} \chi_{\left[a_{i}+(j-1) r, a_{i}+j r\right]}$ a.e. As the indicator functions $\chi_{\left[a_{i}+(j-1) r, a_{i}+j r\right]}$ are translates of the indicator function on $[0, r]$, we have $\chi_{\left[a_{i}+(j-1) r, a_{i}+j r\right]} \in$ $\operatorname{LST}\left(\chi_{[0, r]}\right)$. Hence $g_{i}=\sum_{j=1}^{m_{i}} \chi_{\left[a_{i}+(j-1) r, a_{i}+j r\right]} \in \operatorname{LST}\left(\chi_{[0, r]}\right)$. Therefore $g=\sum_{i=1}^{n} c_{i} g_{i} \in \operatorname{LST}\left(\chi_{[0, r]}\right)$ and hence $\mu=\sum_{i=1}^{n} c_{i} \chi_{\left[a_{i}, b_{i}\right]}=g$ a.e.

(ii) In the above proof,

$$
\begin{aligned}
g_{i} & =\sum_{j=1}^{m_{i}} \chi_{\left[a_{i}+(j-1) r, a_{i}+j r\right]} \\
& =\sum_{j=1}^{m_{i}} \chi_{[0, r]} \star \delta_{a_{i}+(j-1) r} \\
& =\chi_{[0, r]} \star\left(\sum_{j=1}^{m_{i}} \delta_{a_{i}+(j-1) r}\right) \\
& =\chi_{[0, r]} \star v_{i},
\end{aligned}
$$


where $v_{i}=\sum_{j=1}^{m_{i}} \delta_{a_{i}+(j-1) r} \in M_{c d}(\mathbb{R})$. But $g=\sum_{i=1}^{n} c_{i} g_{i}$ a.e. Therefore

$$
\begin{aligned}
g & =\sum_{i=1}^{n} c_{i} g_{i} \\
& =\sum_{i=1}^{n} \chi_{[0, r]} \star c_{i} v_{i} \\
& =\chi_{[0, r]} \star\left(\sum_{i=1}^{n} c_{i} v_{i}\right) \\
& =\chi_{[0, r]} \star v,
\end{aligned}
$$

where $v=\sum_{i=1}^{n} c_{i} v_{i} \in M_{c d}(\mathbb{R})$. Since $\mu=g$ a.e, we have $\mu=\chi_{[0, r]} \star v$ a.e.

Also we have

$$
f \star \chi_{\left[a_{i}, b_{i}\right]}(x)=\int_{a_{i}}^{b_{i}} f(x-y) d y=\sum_{j=1}^{m_{i}} \int_{a_{i}+(j-1) r}^{a_{i}+j r} f(x-y) d y=\left(f \star g_{i}\right)(x) .
$$

Therefore $f \star \mu=f \star g=f \star \chi_{[0, r]} \star v$.

The first part of the following theorem is an extension of [4] and the second part is a simple extension of [14].

Theorem 2.1. (i) For every $g \in C^{r}(\mathbb{R})$ and every $v \in M_{c d}(\mathbb{R})$ with $v \neq 0$ there exists $f \in C^{r}(\mathbb{R})$ such that $f \star v=g$.

(ii) For every $g \in C^{r+1}(\mathbb{R})$, there exists $f \in C^{r}(\mathbb{R})$ such that $f \star \chi_{[a, b]}=g$.

Proof. For $\epsilon>0$, choose $\phi_{\epsilon} \in C^{r+1}(\mathbb{R})$ such that $\operatorname{supp}\left(\phi_{\epsilon}\right) \subset(-\epsilon, \epsilon)$ and $\int_{-\epsilon}^{\epsilon} \phi_{\epsilon}(x) d x=1$. Define $\eta_{1}, \eta_{2} \in C(\mathbb{R})$ by

$$
\begin{aligned}
& \eta_{1}(x):= \begin{cases}0 & \text { if } x \leq-k \\
\frac{x+k}{2 k} & \text { if }-k \leq x \leq k \\
1 & \text { if } x \geq k\end{cases} \\
& \eta_{2}(x):= \begin{cases}1 & \text { if } x \leq-k \\
\frac{k-x}{2 k} & \text { if }-k \leq x \leq k . \\
0 & \text { if } x \geq k\end{cases}
\end{aligned}
$$

It is simple to check that $\eta_{1}(x)+\eta_{2}(x)=1$ for all $x \in \mathbb{R}$. Convolving both sides with $\phi_{\epsilon}$, we get $\left(\eta_{1} \star \phi_{\epsilon}\right)(x)+\left(\eta_{2} \star \phi_{\epsilon}\right)(x)=1$ for all $x \in \mathbb{R}$. Define

$$
g_{1}(x)=g(x)\left(\eta_{1} \star \phi_{\epsilon}\right)(x) \text { and } g_{2}(x)=g(x)\left(\eta_{2} \star \phi_{\epsilon}\right)(x) .
$$

Then $g_{1}, g_{2} \in C^{r+1}(\mathbb{R})$ and $\operatorname{supp}\left(g_{1}\right) \subset[-k-2 \epsilon, \infty)$ and $\operatorname{supp}\left(g_{2}\right) \subset(-\infty, k+2 \epsilon]$. Also we have $g_{1}+g_{2}=g$. 
(i) We show that $f_{1} \star \mu=g_{1}$ and $f_{2} \star \mu=g_{2}$ have solutions $f_{1}$ and $f_{2}$ respectively in $C^{r}(\mathbb{R})$. These $f_{1}$ and $f_{2}$ are then used to construct a solution of the equation $f \star \mu=g$.

$$
\text { Let } \mu=\sum_{i=1}^{n} c_{i} \delta_{x_{i}}, x_{i_{0}}=\operatorname{Min}\left\{x_{1}, x_{2}, \ldots, x_{n}\right\} \text { and } x_{j_{0}}=\operatorname{Max}\left\{x_{1}, x_{2}, \ldots, x_{n}\right\} \text {. }
$$

We can write

$$
\begin{aligned}
\mu & =\sum_{i=1}^{n} c_{i} \delta_{x_{i}} \\
& =\sum_{i=1}^{n} c_{i} \delta_{x_{i}} \star \delta_{-x_{i_{0}}} \star \delta_{x_{i_{0}}} \\
& =\delta_{x_{i_{0}}} \star \sum_{i=1}^{n} c_{i} \delta_{x_{i}-x_{i_{0}}} \\
& =c_{i_{0}} \delta_{x_{i_{0}}} \star\left(\delta_{0}+\sum_{i=1, i \neq i_{0}}^{n} \frac{c_{i}}{c_{i_{0}}} \delta_{x_{i}-x_{i_{0}}}\right) \\
& =c_{i_{0}} \delta_{x_{i_{0}}} \star\left(\delta_{0}+v\right), \quad \text { where } v=\sum_{i=1, i \neq i_{0}}^{n} \frac{c_{i}}{c_{i_{0}}} \delta_{x_{i}-x_{i_{0}}} .
\end{aligned}
$$

Also we can write $\mu$ as

$$
\begin{aligned}
\mu & =\sum_{j=1}^{n} c_{j} \delta_{x_{j}} \\
& =\sum_{j=1}^{n} c_{j} \delta_{x_{j}} \star \delta_{-x_{j_{0}}} \star \delta_{x_{j_{0}}} \\
& =\delta_{x_{j_{0}}} \star \sum_{j=1}^{n} c_{j} \delta_{x_{j}-x_{j_{0}}} \\
& =c_{j_{0}} \delta_{x_{j_{0}}} \star\left(\delta_{0}+\sum_{j=1, j \neq j_{0}}^{n} \frac{c_{j}}{c_{j_{0}}} \delta_{x_{j}-x_{j_{0}}}\right) \\
& =c_{j_{0}} \delta_{x_{j_{0}}} \star\left(\delta_{0}+\psi\right), \quad \text { where } \psi=\sum_{j=1, j \neq j_{0}}^{n} \frac{c_{j}}{c_{j_{0}}} \delta_{x_{j}-x_{j_{0}}} .
\end{aligned}
$$

Define $\alpha=\frac{1}{2} \min \left\{x_{i}-x_{i_{0}} / 1 \leq i \leq n, i \neq i_{0}\right\}$ and $\beta=\frac{1}{2} \min \left\{x_{j_{0}}-x_{j} / 1 \leq j \leq n, j \neq j_{0}\right\}$.

Then $x_{i}-x_{i_{0}}>\alpha, x_{j}-x_{j_{0}}<-\beta$. Hence $\operatorname{supp}(v) \subset(\alpha, \infty)$ and $\operatorname{supp}(\psi) \subset(-\infty,-\beta)$.

Using lemma 1 , we get $h_{1}, h_{2} \in C^{r}(\mathbb{R})$ such that

$$
h_{1} *\left(\delta_{o}+v\right)=g_{1}
$$

and

$$
h_{2} *\left(\delta_{o}+\psi\right)=g_{2}
$$

Convolving both sides of the equation (4) with $c_{i_{0}} \delta_{x_{i_{0}}}$ and the equation (5) with $c_{j_{0}} \delta_{x_{j_{0}}}$, we get $h_{1} *\left(\delta_{o}+v\right) \star c_{i_{0}} \delta_{x_{i_{0}}}=c_{i_{0}} g_{1} \star \delta_{x_{i_{0}}}$ and $h_{2} *\left(\delta_{o}+\psi\right) \star c_{j_{0}} \delta_{x_{j_{0}}}=c_{j_{0}} g_{2} \star \delta_{x_{j_{0}}}$. That is

$$
h_{1} * \mu=c_{i_{0}} g_{1} \star \delta_{x_{i_{0}}}
$$


and

$$
h_{2} * \mu=c_{j_{0}} g_{2} \star \delta_{x_{j_{0}}} .
$$

Equations (6) and (7) imply $\left(\frac{1}{c_{i_{0}}} h_{1} \star \delta_{-x_{i_{0}}}\right) \star \mu=g_{1}$ and $\left(\frac{1}{c_{j_{0}}} h_{2} \star \delta_{-x_{j_{0}}}\right) \star \mu=g_{2}$.

Define $f=\frac{1}{c_{i_{0}}} h_{1} \star \delta_{-x_{i_{0}}}+\frac{1}{c_{j_{0}}} h_{2} \star \delta_{-x_{j_{0}}}$. Then $f \in C^{r}(\mathbb{R})$. Now

$$
\begin{aligned}
f \star \mu & =\left(\frac{1}{c_{i_{0}}} h_{1} \star \delta_{-x_{i_{0}}}\right) \star \mu+\left(\frac{1}{c_{n}} h_{2} \star \delta_{-x_{j_{0}}}\right) \star \mu \\
& =g_{1}+g_{2} \\
& =g .
\end{aligned}
$$

(ii) Using Lemma 2, we get $f_{1}, f_{2} \in C^{r}(\mathbb{R})$ such that $f_{1} \star \chi_{[a, b]}=g_{1}$ and $f_{2} \star \chi_{[a, b]}=g_{2}$. Then $f=f_{1}+f_{2} \in C^{r}(\mathbb{R})$ will satisfy $f \star \chi_{[a, b]}=g$.

Theorem 2.2. For $g \in C^{r+1}(\mathbb{R})$, the following hold:

(i) For every $\mu \in \operatorname{LST}\left(\chi_{[a, b]}\right)$ with $\mu \neq 0$ a.e., there exists $f \in C^{r}(\mathbb{R})$ such that $f \star \mu=g$.

(ii) If there exists $r \in \mathbb{R}$ such that $\frac{b_{i}-a_{i}}{r} \in \mathbb{Z}$ and $\mu=\sum_{i=1}^{n} c_{i} \chi_{\left[a_{i}, b_{i}\right]} \neq 0$ a.e., then there exists $f \in C^{r}(\mathbb{R})$ such that $f \star \mu=g$.

Proof. (i) By lemma 3, there exists $v \in M_{c d}(\mathbb{R})$ such that $\mu=\chi_{[a, b]} \star v$. Applying Theorem 2.1, we get a $h \in C^{r+1}(\mathbb{R})$ and $f \in C^{r}(\mathbb{R})$ such that $h \star v=g$ and $f \star \chi_{[a, b]}=h$. It is simple to verify that $f \star \mu=g$.

(ii) Using Lemma 3, we can write $\mu=\chi_{[a, b]} \star v$ a.e for some $v \in M_{c d}(\mathbb{R})$. As in previous part we obtain $f \in C^{r}(\mathbb{R})$ such that $f \star \mu=g$.

Remark 1. The operator $T_{\mu}$ defined by $T_{\mu}(f)=f \star \mu$ is $1-1$ if we restrict the domain of $T_{\mu}$ to the space of integrable functions $L_{1}(\mathbb{R})$. This can be seen as follows:Suppose $f \star \mu=0$ and $f \in L_{1}(\mathbb{R})$. Since $f$ is integrable and $\mu$ is compactly supported, the Fourier transforms of both $f$ and $\mu$ namely $\hat{f}$ and $\hat{\mu}$ are holomorphic on $\mathbb{C}$. Hence the corresponding zero sets $z(\hat{f})$ and $z(\hat{\mu})$ are of measure zero. Therefore we get $f=0$ a.e.

Remark 2. When $\mu \in \operatorname{LST}\left(\chi_{[a, b]}\right)$ or $\mu=g$ a.e for some $g \in \operatorname{LST}\left(\chi_{[a, b]}\right)$, the kernel of the operator $T_{\mu}$ is a nontrivial subspace of $C(\mathbb{R})$. For, since $\mu$ can be written as $\mu=\chi_{[0, r]} \star v$ for some $v \in M_{c d}(\mathbb{R})$. This implies that $\lambda=\frac{2 n \pi}{r} \in z(\hat{\mu})$ for $n \in \mathbb{Z}$. Therefore $e^{i \lambda x} \in \operatorname{Ker}\left(T_{\mu}\right)$. Hence there are infinitely many solutions to the convolution equation $f \star \mu=g$.

Remark 3. Theorem 2.2 is possible even if $g \in L_{1}(\mathbb{R})$ with $\hat{g}(\lambda) \neq 0$ and the Fourier-Laplace transform $\hat{\mu}(\lambda)=0$ for some $\lambda \in \mathbb{C}$. 


\section{References}

[1] S. C. Bagchi and A. Sitaram, Spherical mean-periodic functions on semi simple Lie groups, Pacific J. Math., 84(1979), 241-250.

[2] C. A. Berenstein and B. A. Taylor, Mean-periodic functions, Internat. J. Math. \& Math. Sci., 3(1980), $199-235$.

[3] J. Delsarte, Les fonctions moyenne-periodiques, J. Math. Pures Appl., 14(1935), 403-453.

[4] P. Devaraj, Reconstruction from local averages involving discrete measures, Rendiconti del Circolo Matematico di Palermo, 59(2010), 261-166.

[5] P. Devaraj and Inder K. Rana, Vector valued mean-periodic functions, Journal of Australian Mathematical Society, 72(2002), 363-388.

[6] G. A. Edgar and J. M. Rosenblatt, Difference equations over locally compact abelain groups, Transactions of the American Mathematical Society, 253(1979), 273-289.

[7] L. Ehrenpreis, Appendix to the paper "Mean periodic functions I", Amer. J. Math., 77(1955), 731-733.

[8] L. Ehrenpreis, Mean periodic functions, Part I. Varieties whose annihilator ideals are principal, Amer. J. Math., 77(1955), 293-328.

[9] L. Ehrenpreis, Solutions of some problems of division III, Amer. J. Math., 78(1956), 685-715.

[10] F. John, Continuous dependence on data for solutions of partial differential equations with prescribed bound, Comm. Pure Appl. Math., 13(1960), 551-585.

[11] L. Hörmander, On the range of convolution operators, Annals of Mathematics 76(1968), 148-169.

[12] J. P. Kahane, Lectures on mean-periodic functions, Tata Institute, 1957.

[13] B. Malgrange, Existence et approximation des solutions des equations aux derivees partielles et des equations de convolutions, Ann. Inst. Fourier (Grenoble) 6(1955-56), 271-355.

[14] E. Novak and Inder K. Rana, On the unsmoothing of functions on the real line, Proc. Nede. Acad.Sci. Ser. A 89(1986), 201-207.

[15] B. van der Pol, Smoothing and Unsmoothing, In: M. Kac, Probability and related topics in physical sciences. New York: Interscience 1959.

[16] B. van der Pol and H. Bremmer, Operational Calculus, Cambridge University Press, 1964.

[17] Inder K. Rana, Unsmoothing over balls via plane wave decomposition, Rend. Cir. Mat. Palermo 2(34)(1990), 217-234.

[18] L. Schwartz, Theorie generale des fonctions moyenne-periodiquies, Ann. of Math., 48(1947), 857-929.

[19] S. Thangavelu, Mean periodic functions on phase space and the Pompeiu problem with a twist, Ann. Inst. Fourier (Grenoble) 45(1995), 1007-1035.

[20] Y. Weit, On Schwartz theorem for the motion group, Ann. Inst. Fourier (Grenoble), 30(1980), 91-107.

[21] L. Szekelyhidi, Spectral synthesis problem on locally compact groups, Monatsh Math, Springer-Verlag, 2009.

Department of Mathematics, College of Engineering Guindy, Anna University Chennai, India, PIN 600025.

E-mail: devaraj@annauniv.edu 